\title{
Análisis de los recursos locales de Servicios Sociales destinados a las familias en situación de riesgo psicosocial en un contexto de crisis
}

\author{
Susana Martí-García ${ }^{1}$; Octavio Vázquez-Aguado²; Manuela Ángela Fernández-Borrero ${ }^{3}$
}

Recibido: 22/03/2017 / Revisado: 19/04/2017 / Aceptado: 31/05/2018

Disponible on line

Resumen. En este artículo se analizan los recursos locales de Servicios Sociales comunitarios puestos a disposición de familias que tienen menores a cargo y que atraviesan dificultades. Los objetivos abordan, por un lado, el estudio de las características principales de estas acciones, especialmente las de carácter psicoeducativo. Por otro lado, se ponen en relación los recursos de intervención con las zonas básicas de Servicios Sociales (ZBSS) a las que van dirigidos. Siguiendo una metodología particularmente cuantitativa, los resultados principales giran en torno a la homogeneidad de actuaciones en toda la provincia de Huelva y a la necesidad de derivación para acceder a las mismas, en la mayoría de los casos. También se destaca la importancia de las intervenciones psicoeducativas que, junto a los recursos de carácter económico, tienen una mayor implementación que las demás actuaciones. Entre las principales conclusiones se encuentran: la necesidad de la acción integral en los ejes económico, interrelacional y psicosocial; la importancia de las evaluaciones que generen intervenciones basadas en la evidencia; y el contexto de intervención local como pieza clave en la creación de políticas sociales más próximas y, por tanto, más reales y eficientes

Palabras clave: Servicios Sociales; Estado de bienestar; intervención social; familia; crisis.

\section{[en] Analysis of local social services resources allocated to families in situations of psychosocial risk within a crisis context}

\begin{abstract}
This article contains an analysis of the local community social services resources made available to families that are responsible for minors and experiencing difficulties. The first aim of the article is to study the main characteristics of these actions and particularly those of a psychoeducational nature. The second aim is to relate the intervention resources and basic areas of social services to their recipients. Based on an essentially quantitative methodology, the main results concern the homogeneity of actions throughout the province of Huelva and the need for referral in order to access these actions in the majority of cases. Also of note is the importance of psychoeducational interventions, which together with economics resources are implemented to a greater degree in comparison with other actions. The main conclusions include a need for comprehensive action in financial, inter-relational and psychosocial contexts, the importance of evaluations that lead to evidence-based interventions, and the key role of the local intervention context in the creation of social policies that are more immediate, and hence more real and efficient.
\end{abstract}

Key words: social services; Welfare State; social intervention; family; crisis.

Sumario: Introducción. 1. Método. 2. Instrumentos. 3. Participantes. 4. Resultados. 5. Discusión. 6. Conclusiones. 7. Referencias bibliográficas.

Cómo citar: Martí-García, S.; Vázquez-Aguado, O.; Fernández-Borrero, M.Á. (2019). Análisis de los recursos locales de Servicios Sociales destinados a las familias en situación de riesgo psicosocial en un contexto de crisis, en Cuad. trab. soc. 32(1), 85-98.

Universidad de Huelva, España susana.marti@dstso.uhu.es

Universidad de Huelva, España octavio@uhu.es

3 Universidad de Huelva, España manuela.fernandez@dstso.uhu.es 


\section{Introducción}

Los Servicios Sociales comunitarios constituyen en la actualidad la red básica de soporte para muchos individuos y familias. Para el caso de Andalucía, según las estadísticas oficiales de la Junta de Andalucía, en torno a 1.000.000 de personas acuden anualmente a este dispositivo. El actual período de crisis socioeconómica, acompañado del cambio de múltiples factores y de la aparición de diversos fenómenos emergentes, ha incidido en el incremento de la heterogeneidad de las necesidades sociales y en el aumento de las situaciones familiares que pueden ser consideradas de riesgo por encontrarse en estadios de vulnerabilidad y de exclusión social (Garcés, 2012).

En este sentido tampoco hay que olvidar los innumerables y profundos efectos que la crisis ha provocado en la infancia y sus condiciones de vida, especialmente en nuestro país. Condiciones íntimamente ligadas a la desigualdad social tanto actual como futura y que no han sido objeto de respuestas categóricas por parte del ámbito social público. Más bien, se puede decir que la disminución de los recursos disponibles dedicados a la infancia ha sido significativa (Navarro y Clua-Losada, 2012).

Los Servicios Sociales constituyen un sistema de protección y prevención fundamental que, según Casado (2008), se integran como instrumento de la política social puesta en marcha por la sociedad y los poderes públicos para atender las necesidades básicas de los individuos, familias, grupos y comunidades.

No obstante, la acción pública también ha sido objeto de transformaciones coyunturales de diversa índole, enmarcadas dentro de las estrictas exigencias del mercado y de la ideología neoliberal. Esta circunstancia se ha traducido en intervenciones sociales que retornan al pasado terreno moral (Hamzaoui, 2005). Es en este punto donde, como dice Garcés (2012), la percepción económica toma un protagonismo excesivo y el Estado de bienestar vuelve a desempeñar su papel primigenio en respuesta a la amenazadora inestabilidad de su función actual.

El desarrollo normativo del Sistema público de Servicios Sociales se inicia dentro del marco legislativo de la Constitución Española de 1978, que señala en su artículo 148.1.20 la competencia en materia de asistencia social de las distintas comunidades autónomas. También se vincula al artículo 13.22 del primer Es- tatuto andaluz de 1981, que ratificaba la competencia en Servicios Sociales otorgada por nuestro texto constitucional. Así, en Andalucía nace la primera Ley de Servicios Sociales (Ley 2/1988, de 4 de abril, de Servicios Sociales de Andalucía), vigente casi tres décadas y derogada por la reciente Ley 9/2016, de 27 de diciembre, de Servicios Sociales de Andalucía. La ratificación de la competencia autonómica en esta materia también se contempla en el actual Estatuto de Autonomía de Andalucía (Ley Orgánica 2/2007, de 19 de marzo, de Reforma del Estatuto de Autonomía de Andalucía) en su artículo 61.1.a.

La nueva Ley andaluza de Servicios Sociales define el Sistema Público de Servicios Sociales como una red integrada de responsabilidad y control público de atención con una función promotora, preventiva, protectora $\mathrm{y}$ asistencial (art.2.5). Se trata de un sistema de carácter universal (art. 7.1), suponiendo un derecho subjetivo y exigible (art. 3.6 y art. 7.2). $\mathrm{Su}$ finalidad natural se dirige hacia el impulso de la acción social para conseguir el bienestar desde el empoderamiento, la autonomía y la autodeterminación individual y colectiva. Todo ello en base a un trabajo cooperativo entre ciudadanía, agentes económicos, sociales e instituciones (art. 24.4).

El Sistema Público de Servicios Sociales se estructura en el primer nivel de atención en los Servicios Sociales comunitarios. Éstos constituyen la estructura básica de servicios sociales y el acceso al resto de los dispositivos asistenciales que componen la estructura del sistema. Además, son la primera referencia en cuanto al proceso de intervención en base a las necesidades (valoración, planificación, tratamiento, seguimiento y coordinación) y la prevención de situaciones de vulnerabilidad social. Sus características intrínsecas más destacadas están relacionadas con la universalidad y con la proximidad a la comunidad (art. 26 y art. 27).

\section{La familia. Realidad dinámica, objeto y sujeto de intervención social}

La familia, en palabras de Rodrigo y Palacios (1998), se puede definir como:

La unión de personas que comparten un proyecto vital de existencia en común que se quiere duradero, en el que se generan fuertes sentimientos de pertenencia a dicho grupo, existe 
un compromiso personal entre sus miembros y se establecen intensas relaciones de intimidad, reciprocidad y dependencia (p. 33).

A esta conceptualización se añaden cuestiones estructurales que tienen que ver con la importancia de las unidades familiares en los procesos de socialización y reproducción de los sistemas normativos, así como el cuidado y la protección de los miembros que la integran.

En los países que pertenecen al modelo mediterráneo de bienestar, como es el caso de España, la familia juega un rol clave en los procesos sociales, complementando y apoyando en numerosas ocasiones al propio sistema al actuar como pilar sustentador del individuo y, por tanto, de la sociedad. Este hecho constituye un elemento característico y diferenciador del resto de modelos (Moreno, 2002; Flaquer, 2004; Flaquer, 2006; Montagut, 2008).

El contexto familiar tiene un papel fundamental para un desarrollo humano saludable. Supone la unidad básica de integración social y, por lo tanto, la base educativa de la trayectoria individual y social a lo largo de todo el ciclo vital, con una vinculación afectiva segura y estimulante. Éste será un proceso en constante evolución a lo largo de toda la vida de la persona, donde el sistema familiar siempre estará presente y activo en su función (Rodrigo y Palacios, 1998).

Este posicionamiento de la familia como agente clave en el bienestar de las personas y sociedades está experimentando cambios relevantes en los últimos tiempos, derivados del incremento de diversidad de situaciones y familias, así como de adaptaciones que tienen que realizarse para dar respuesta a las situaciones de dificultad que muchas familias viven como resultado del proceso de recesión y crisis. Todo ello genera nuevas ramificaciones tanto en la forma como en el funcionamiento de este sistema social, que en ocasiones han traído asociados fenómenos de riesgo social que empujan consigo a las familias hacia procesos de vulnerabilidad y situaciones de exclusión social.

Estamos ante lo que se denomina posmodernización familiar, como asegura Beck (1996), una modernización reflexiva que pretende significar una autotransformación y que se refleja en un aumento en la diversidad y complejidad de situaciones de necesidad y carencia.

La pluralidad de problemáticas familiares, fruto de la evolución social y de las caracte- rísticas propias de un sistema de bienestar mediterráneo, se encuentra vinculada con el actual panorama de recesión económica sistémica. En este escenario concurre el deterioro de los Servicios Sociales en la mayoría de las comunidades autónomas españolas (11 de las 17 existentes no llegan al nivel de desarrollo medio bajo). Así lo expresa el último Informe sobre el Indice de Desarrollo de los Servicios Sociales (DEC) correspondiente al año 2015 (García, Barriga, Ramírez, Zubiría y Velasco, 2016). Entre ellas y en décimo lugar se sitúa Andalucía, con un irrelevante nivel de desarrollo de Servicios Sociales, aun contando con una mejora en esta evolución con respecto al año anterior.

Esta perspectiva de recesión del sistema es ratificada en el II Informe sobre los Servicios Sociales en España (Lima, 2016), a partir de los discursos profesionales sobre la gestión de la crisis económica, llevada a cabo desde los poderes públicos. Los resultados de este Informe muestran un amplio consenso entre los trabajadores y las trabajadoras sociales en afirmar que las medidas de austeridad, lejos de mitigar las necesidades de las personas y familias en situación de vulnerabilidad, las ha agravado de forma directa y contundente, sin detenerse en analizar el impacto de estas políticas en los servicios sociales, en las personas que éstos atienden y, en definitiva, en la cohesión y el bienestar social.

La presencia de situaciones adversas hace que existan muchos casos en los que los sistemas familiares no pueden llevar a cabo sus cometidos con respecto a los niños, niñas y adolescentes; ya sea bien de forma puntual o permanente y siendo además incapaces de solucionar los problemas por sí mismos. Estas son las familias que se denominan en situación de riesgo psicosocial y son, por lo tanto, disfuncionales (Rodrigo, Máiquez, Martín y Byrne, 2008).

En este sentido y en línea con las circunstancias adversas familiares, se encuentra el auge de las intervenciones familiares bajo el concepto de preservación familiar y promoción de la parentalidad positiva. Aquí es donde se encuadran las llamadas intervenciones psicoeducativas, que buscan apoyar y facilitar el buen ejercicio de la responsabilidad familiar, dando respuesta a las situaciones de riesgo con un enfoque positivo, diverso y amplio de la familia, de sus transacciones y crisis y de la búsqueda de respuestas específicas en cada caso 
concreto. Todo ello mediante actuaciones que posibilitan el desarrollo de habilidades y capacidades de afrontamiento y optimización funcional, una alternativa a la ya clásica finalidad reparadora o de tratamiento que descansa en la teoría del déficit y que desemboca en medidas de naturaleza punitiva (Rodrigo, Máiquez, Martín y Byrne, 2008; Rodrigo, Máiquez y Martín-Quintana, 2010; Menéndez, Arenas, Pérez y Lorence, 2012).

Tanto estas intervenciones psicoeducativas como el resto de actuaciones realizadas por los dispositivos de Servicios Sociales comunitarios se pueden clasificar, atendiendo a la propuesta de Gordon (1980), en tres tipos: universales (toda la población), selectivas (personas, familias o grupos con ciertos requisitos, de nivel de riesgo leve o moderado o que tengan una necesidad o problemática que no implique una situación de adversidad) e indicadas (precisan de valoración y derivación profesional previa y su nivel de riesgo es alto).

\section{Realidad y necesidades de los sistemas familiares en el contexto actual}

Las familias que se sitúan en los niveles mencionados de mayor riesgo o vulnerabilidad, así como aquellas que se pueden considerar en situación de exclusión social, presentan diversas necesidades de diferente naturaleza, en la mayoría de los casos interconectadas. Existen factores presentes en el nivel socioeconómico que generan consecuencias asociadas al "modelo del estrés económico familiar", y que inciden de forma directa "en el ajuste parental y en la calidad de la crianza, los cuales repercuten en variables de ajuste en el adolescente" (Taylor, 2007, en Arranz y Oliva, 2010, p.24).

Los últimos datos de la Encuesta de Condiciones de Vida (ECV, INE, 2016) sitúan, por un lado, la tasa de riesgo de pobreza del conjunto de la población en el 22,1 por ciento, encontrándose los menores de 16 años 6.7 puntos por encima (ECV del año 2015). En cuanto al tipo de hogar, la mayor tasa de riesgo de pobreza se asienta en los hogares donde conviven menores (37,5 por ciento). Como dato a destacar, Andalucía se sitúa en el primer puesto a nivel autonómico con un 35,7 por ciento de tasa de riesgo de pobreza.

Por otro lado, los ingresos medios anuales de los hogares españoles se han reducido el 0,2 por ciento respecto al año anterior que, aunque en menor medida, siguen la tónica descendente desde que comenzó la crisis en 2008. Según reflejan los datos sobre la situación y las dificultades económicas de los hogares, el 13,7 por ciento de los encuestados reconoció tener mucha dificultad para llegar a fin de mes. Los hogares andaluces se presentan entre los porcentajes más elevados en relación con la capacidad de afrontar gastos imprevistos $(55,0$ por ciento) y permitirse ir de vacaciones al menos una semana al año ( 55,0 por ciento).

No obstante, cabe destacar la reducción que la tasa AROPE ha experimentado en sus tres ejes de análisis en 2015: riesgo de pobreza $(0,1$ por ciento), carencia material severa $(0,7$ por ciento) y baja intensidad en el empleo (1,7 por ciento).

Otro aspecto a tener en cuenta en el ámbito de las necesidades familiares es la falta de empleo. Según el Decreto-Ley 8/2014, de 10 de junio, de medidas extraordinarias y urgentes para la inclusión social a través del empleo y el fomento de la solidaridad en Andalucía, el número de familias en situación de exclusión social o en riesgo de estarlo han aumentado considerablemente en Andalucía. Muchas familias, que antes no precisaban la atención de los Servicios Sociales ni tampoco ayudas públicas para cubrir sus necesidades básicas, se ven abocadas a vivir bajo los umbrales de pobreza, intensificándose esta situación en familias con menores a cargo.

Además de las necesidades económicas, las familias presentan necesidades vinculadas a las relaciones y al funcionamiento familiar. La presencia de dificultades de diversa índole puede conllevar conflictos relacionales que inciden en desajustes funcionales. Como dice Ruiz (1999), esta presencia de conflicto crea una atmósfera de inseguridad que dificulta el desarrollo funcional adecuado de la familia y de sus miembros.

En este tipo de necesidades, así como en las socioeconómicas descritas, disponer de un apoyo social es un elemento de protección fundamental. Éste, junto con el capital social, que implica diversidad en la estructura de la red y calidad de las relaciones, es uno de los elementos que favorecen la práctica de la parentalidad positiva (Rodrigo, Máiquez y Martín-Quintana, 2010).

Desde este enfoque resulta relevante la protección de las redes familiares y la potenciación y el fortalecimiento de una red de apoyo social informal primordial, ya que sirve de 
contención en transiciones normativas naturales familiares y en aquellas extraordinarias que suponen un riesgo inesperado y que ponen a prueba los factores protectores y las habilidades de afrontamiento familiar. Aun así, esta gran malla protectora en ocasiones no es suficiente ante situaciones que desbordan tanto las capacidades familiares como las de sus fuentes de apoyo. Es entonces cuando la red de apoyo formal pasa a formar parte de las herramientas de respuesta ante situaciones de riesgo, con el objetivo de lograr bienestar y unas condiciones de vida óptimas. Cuestiones que son abordadas desde el ámbito público administrativo e institucional, especialmente, desde el Sistema Público de Servicios Sociales.

De acuerdo con ello, el actual Sistema español de protección social intenta garantizar la atención formal a familias con menores y que estén en situación de dificultad, a través de actuaciones y de apoyo familiar que se llevan a cabo desde los dispositivos de Servicios Sociales. Las políticas sociales han activado recursos de apoyo familiar, pero aunque exista respaldo legislativo e institucional, las medidas adoptadas suponen una ayuda insuficiente frente a la considerable acción social que realizan las familias, pues "a menudo el modelo se da por sentado y es por eso por lo que gran parte de las leyes y de las políticas sociales sólo brindan protección al modelo de manera indirecta o implícita" (Naldini, 2003).

En el marco de la realidad familiar descrito, es importante destacar la escasez de investigaciones a nivel local, tanto de estudio de necesidades familiares como de análisis de los recursos sociales individuales y comunitarios destinados a las mismas. Los dispositivos locales son los que están más próximos a las familias, conocen las situaciones de necesidad de primera mano, realizan los diagnósticos pertinentes y los resultados de sus decisiones y acciones recaen directamente en sobre ellas.

Tener conocimiento del saber profesional desarrollado en el área de servicios sociales municipales permite discernir mejor las demandas que pueden estar generándose y que no se pueden estar teniendo en cuenta en el diseño y evaluación de las actuaciones de mayor nivel como pueden ser planes y programas. Acciones que deben basarse en políticas innovadoras que respondan a las nuevas necesidades sociales en cada contexto (De la Red y Barranco, 2014).

Este artículo tiene como finalidad presentar la realidad de los recursos de atención a las familias que se prestan desde los Servicios Sociales comunitarios, desde la base del conocimiento inicial de los recursos disponibles en el caso de Huelva.

La necesidad de conocer las intervenciones y recursos es algo que ya se ha puesto de manifiesto previamente. Este trabajo aborda, por un lado, el estudio de las intervenciones de atención y apoyo familiar que se implementan desde los dispositivos pertenecientes a los Servicios Sociales comunitarios y, por otro lado, se pretende analizar en qué medida los recursos pueden estar relacionados con las zonas básicas de Servicios Sociales (ZBSS). La población diana a la que van dirigidas estas actuaciones serán familias con hijos menores a cargo y que se encuentren en una situación de vulnerabilidad social, según las diversas necesidades presentes en el sistema familiar.

La difusión de los resultados del presente estudio ha contado con el apoyo del Ministerio de Educación, Cultura y Deporte del Gobierno de España a través del Programa Nacional de Formación de Profesorado Universitario (FPU15/03909).

\section{Método}

La metodología del trabajo ha sido fundamentalmente cuantitativa. Se plantean los siguientes objetivos específicos:

1. Examinar las características principales de los recursos de intervención y apoyo familiar.

2. Analizar las relaciones que pueden existir entre las características de los recursos y las zonas a las que van destinados.

3. Explorar las características y condiciones específicas de los programas de carácter psicoeducativo.

\section{Participantes}

En el estudio participaron 15 dispositivos pertenecientes al nivel básico de atención del Sistema Público de Servicios Sociales onubense. Nueve de ellos pertenecían a las zonas básicas de Servicios Sociales de la Diputación Provincial de Huelva, que dan servicio a un total de 73 municipios. Los seis restantes correspondían a los Ayuntamientos de más de 20.000 habitantes y, por ello, independientes en materia de coordinación y gestión de sus Servicios Sociales Comunitarios. En la tabla 
1 se muestran los datos descriptivos principales de los dispositivos de los Servicios Socia- les Comunitarios pertenecientes a las Corporaciones Locales.

Tabla 1. Descripción básica dispositivos de Servicios Sociales en corporaciones locales onubenses

\begin{tabular}{|c|c|c|}
\hline ZBSS* & Núm. municipios/núcleos población & Núm. UTS \\
\hline \multicolumn{3}{|l|}{ Diputación } \\
\hline Andévalo & 13 & 6 \\
\hline Cinturón Agroindustrial & 5 & 5 \\
\hline Condado Norte & 6 & 4 \\
\hline Condado Sur & 5 & 5 \\
\hline Costa & 3 & 4 \\
\hline Cuenca Minera** & 8 & 5 \\
\hline Rivera del Tinto & 4 & 4 \\
\hline Sierra Este & 17 & 6 \\
\hline Sierra Oeste & 12 & 5 \\
\hline \multicolumn{3}{|l|}{ Ayuntamientos } \\
\hline Almonte & 5 & 1 \\
\hline Ayamonte & 7 & 1 \\
\hline Huelva capital $* * *$ & 1 & 6 \\
\hline Isla Cristina & 9 & 1 \\
\hline Lepe & 6 & 1 \\
\hline Moguer & 4 & 1 \\
\hline
\end{tabular}

Fuente: Elaboración propia a partir de los datos recogidos en el trabajo de campo de la investigación. Nota: ** Zona con Necesidades de Transformación Social (ZNTS). *** ZNTS en tres de sus seis UTS.

\section{Instrumentos}

En el trabajo de campo, realizado en el año 2015, se han utilizado dos herramientas ad hoc de recogida de datos: ficha del recurso y ficha del dispositivo y zona. Las dimensiones y variables de ambas quedan sintetizadas en los cuadros 1 y 2 que siguen a continuación.

\footnotetext{
Sistematicidad: intervenciones sistemáticas cuando han sido realizadas a partir de directrices y evaluaciones en relación a una fundamentación de las necesidades detectadas en la población. Generalmente por imperativo legal, con muchos años de implementación y de carácter común en todas las ZBSS. Parcialmente sistemáticas, aquellas acciones que tienen objetivos y actividades claras y descritas en un documento, pero carecen de un marco teórico, de un estudio de necesidades previo o de evaluaciones que le otorguen un carácter basado en la evidencia. No sistemáticos, tienen objetivos muy generales establecidos, no siguen una metodología y actividades prefijadas, sino que se amoldan a las características de los destinatarios o a cuestiones económicas para su realización.

** Disposición profesional hacia la acción: razones profesionales que han impulsado la implementación.
} 
Cuadro 1. Dimensiones Ficha de Análisis de los Recursos de Intervención y Apoyo Familiar

\begin{tabular}{|c|c|}
\hline Dimensión & Variables \\
\hline $\begin{array}{l}\text { Dispositivo que realiza la in- } \\
\text { tervención }\end{array}$ & $\begin{array}{l}\text { Nombre dispositivo } \\
\text { Nombre recurso } \\
\text { Tipo de recurso (común o específico) } \\
\text { Ámbito territorial actuación } \\
\text { Sector que abarca la intervención }\end{array}$ \\
\hline $\begin{array}{l}\text { Requisitos de acceso pobla- } \\
\text { ción }\end{array}$ & $\begin{array}{l}\text { Principio Universalidad } \\
\text { Colectivo específico sin requisito previo } \\
\text { Colectivo específico con requisito previo } \\
\text { Clasificación intervención Gordon (1987) }\end{array}$ \\
\hline Destinatarios & Colectivo específico objeto de intervención \\
\hline $\begin{array}{l}\text { Características } \\
\text { intervención }\end{array}$ & $\begin{array}{l}\text { Carácter intervención } \\
\text { Modalidad } \\
\text { Sistematicidad } \\
\text { Carácter implementación } \\
\text { Disposición hacia la acción profesional: Razones de elección o puesta en marcha del recurso }\end{array}$ \\
\hline
\end{tabular}

Cuadro 2. Dimensiones Ficha de Análisis del Dispositivo y su Zona

\begin{tabular}{|c|c|}
\hline Dimensión & Variables \\
\hline Datos dispositivo & $\begin{array}{l}\text { Nombre del dispositivo } \\
\text { Zona territorial de actuación (ZBSS) } \\
\text { Contexto rural o urbano } \\
\text { Núm. UTS }\end{array}$ \\
\hline Datos socioeconómicos zona del dispositivo & $\begin{array}{l}\text { Zona con Necesidades de Transformación Social (ZNTS) } \\
\text { Nivel socioeconómico ZBSS: desempleo }\end{array}$ \\
\hline Información territorial & $\begin{array}{l}\text { Nombre municipios comprendidos } \\
\text { Núm. municipios } \\
\text { Núm. núcleos población } \\
\text { Centro cabecera, en su caso } \\
\text { Extensión } \mathrm{km}^{2}\end{array}$ \\
\hline Información poblacional & $\begin{array}{l}\text { Núm. Habitantes zona } \\
\text { Núm. Habitantes núcleos } \\
\text { Núm. Habitantes diseminados } \\
\text { Densidad población } \\
\text { Incremento relativo población en diez años (2004-2014) } \\
\text { Edad media población }\end{array}$ \\
\hline Información familiar & $\begin{array}{l}\text { Núm. hogares parejas con hijos menores } \\
\text { Núm. hogares un adulto progenitor con hijos menores } \\
\text { \% Núcleos monoparentales } \\
\text { Núm. hogares madre con hijos menores } \\
\text { Núm. hogares padre con hijos menores } \\
\text { Tamaño medio hogar } \\
\text { \% Hogares todos sus miembros españoles } \\
\text { \% Hogares alguno de sus miembros extranjero } \\
\text { \% Hogares con una única generación presente } \\
\text { Viviendas familiares principales }\end{array}$ \\
\hline Recursos relevantes objeto de análisis & $\begin{array}{l}\text { Nombre recursos } \\
\text { Núm. total recursos a analizar }\end{array}$ \\
\hline
\end{tabular}


La ficha del recurso aborda los objetivos primero y tercero. Para el objetivo segundo, se utiliza la ficha del dispositivo y zona.

La ficha del dispositivo y zona fue cumplimentada con datos del Instituto de Estadística de Andalucía y otras fuentes estadísticas nacionales por la investigadora. La ficha del recurso, con formato cuestionario, fue cumplimentada por los profesionales de los Servicios Sociales comunitarios de la Diputación Provincial.

Las variables, en relación con los recursos, incluidas en el análisis son: intervención de carácter psicosocial/psicoeducativo; requisito de acceso, según clasificación de Gordon (1980); modalidad de la intervención y grado de sistematicidad. Con respecto a las variables de los dispositivos, se han elegido dos: contexto (rural o urbano) y nivel socioeconómico de la zona. Estas variables se han seleccionado con la lógica de la intervención social y ad hoc por el equipo investigador, dada la ausencia de criterios estandarizados y validados a este nivel territorial.

Los programas psicoeducativos se han analizado con relación a: requisitos de acceso de la población a los recursos según clasificación de Gordon (1980), modalidad de la intervención y grado de sistematicidad.

\section{Resultados}

Los resultados de este trabajo se presentan acordes con la estructura planteada en los objetivos.

\subsection{Características principales de los recursos de intervención y apoyo familiar, sus destinatarios y los requisitos de acceso a éstos}

Se han identificado y analizado un total de 278 recursos, de los que 180 son intervenciones generales en ambas corporaciones locales. El resto de recursos, que ascienden a 98, se tratan de actuaciones específicas realizadas en zonas y municipios concretos. La Diputación provincial cuenta con 73 de estas intervenciones específicas (destacando Cuenca Minera con 11) y un total de 25 corresponden al ámbito municipal de los ayuntamientos (destacando Huelva capital con 9); dos zonas con necesidades especiales.
Los dispositivos de Servicios Sociales de base que cuentan con menos recursos (generales y específicos) de atención y apoyo familiar son la zona básica de Servicios Sociales de Almonte (14), seguida de Isla Cristina (15), Moguer (15) y Ayamonte (16). Todas pertenecen a ayuntamientos de más de 20.000 habitantes. Huelva capital (21) presenta diferencias con respecto a las demás zonas. Los dispositivos con mayor número de recursos pertenecen a la Diputación, destacando la zona de necesidades de transformación social (ZNTS) Cuenca Minera (23).

En relación al ámbito territorial de intervención, 180 recursos son provinciales ( 65 por ciento), 72 comarcales ( 26 por ciento) y 26 municipales ( 9 por ciento). En cuanto al sector de intervención, del total de recursos 180 son intersectoriales, se implementan con la colaboración de otros dispositivos externos (65 por ciento) y un total de 98 se desarrollan exclusivamente por los dispositivos de Servicios Sociales comunitarios ( 35 por ciento).

En cuanto a los requisitos de acceso de la población a los distintos recursos familiares, de los 278 analizados, un total de 191 (69 por ciento) requieren algún requisito previo para acceder, generalmente por pertenecer a un colectivo específico (20,5 por ciento) o por cuestiones económicas (16,2 por ciento). Pero el más representativo de estos requisitos es el nivel de riesgo psicosocial alto (44,2 por ciento). En este sentido, haciendo referencia a la clasificación de Gordon (1980), los resultados muestran que el 45 por ciento son intervenciones selectivas, seguidas muy de cerca por las indicadas con el 44 por ciento y, por último, las universales con el 11 por ciento.

Haciendo referencia a los destinatarios de las acciones, predominan las familias (70 por ciento), los adultos (20 por ciento), los adolescentes ( 7,6 por ciento), los menores en general ( 2 por ciento) y sólo los niños ( 0,4 por ciento).

En relación al carácter de la intervención, los recursos de carácter psicosocial/psicoeducativo ( 31 por ciento) y los de carácter económico (21,8 por ciento) son los más representativos. Los menos frecuentes son los que tienen que ver con el ocio y tiempo libre $(0,6$ por ciento). Con valores intermedios (11 por ciento) cabe destacar las intervenciones que tienen que ver con la vivienda y el empleo. También se encuentran los recursos destinados al apoyo en alimentación ( 6,8 por ciento). 
En referencia a los resultados descriptivos de las demás características básicas de la intervención, como se muestra en la tabla 2, lo más significativo es que más de la mitad de los recursos son de carácter individual/familiar (69,4 por ciento), frente a los recursos grupales/comunitarios (30,6 por ciento). Así como la implementación de los recursos de la muestra se lleva a cabo, en su mayoría, en colaboración con profesionales de otros dispositivos externos a los Servicios Sociales comunitarios, alcanzando un total de 180 acciones intersectoriales (64,7 por ciento). Ninguno de los recursos analizados se implementan de forma totalmente externa. Las actuaciones se llevan a cabo mayormente por mandato legislativo o inclusión en planes de acción (son todas las comunes), seguido por el convencimiento profesional por detección de necesidades del contexto.

Tabla 2. Descriptivos características básicas de la intervención

\begin{tabular}{|c|c|c|}
\hline Característica Básica Intervención & Frecuencia & Porcentaje \\
\hline \multicolumn{3}{|l|}{ Modalidad } \\
\hline Individual/Familiar & 193 & $69,4 \%$ \\
\hline Grupal/Comunitario & 85 & $30,6 \%$ \\
\hline \multicolumn{3}{|l|}{ Sistematicidad* } \\
\hline Sí & 219 & $78,8 \%$ \\
\hline No & 6 & $2,2 \%$ \\
\hline Parcialmente & 53 & $19,1 \%$ \\
\hline \multicolumn{3}{|l|}{ Carácter implementación } \\
\hline Sólo por profesionales del dispositivo & 98 & $35,3 \%$ \\
\hline Colaboración con otros dispositivos y profesionales externos & 180 & $64,7 \%$ \\
\hline \multicolumn{3}{|l|}{ Disposición profesional hacia la acción** } \\
\hline Imperativo legal o Planes de Acción Social & 222 & $79,9 \%$ \\
\hline Convencimiento profesional sin evidencia empírica & 40 & $14,4 \%$ \\
\hline Datos de eficacia disponibles & 15 & $5,4 \%$ \\
\hline Razones de accesibilidad/cercanía/conocimiento/oportunidad & 1 & $0,4 \%$ \\
\hline Totales & 353 & $100 \%$ \\
\hline
\end{tabular}

\subsection{Relación entre las características de los recursos destinados a las familias y las zonas de aplicación}

A continuación se detallan los resultados obtenidos en la variable "contexto" con respecto a los cuatro ítems de la ficha de recursos citados en la metodología. En primer lugar, hablaremos de la relación existente entre el contexto rural o urbano y las intervenciones de carácter psicosocial/psicoeducativo. Los análisis estadísticos ponen de manifiesto que no existen diferencias estadísticamente significativas en este tipo de actuaciones en función del contexto de la zona de aplicación $\left(X^{2}=.043 ; p=.836\right)$.

En segundo lugar, con respecto a la relación entre los contextos de intervención y la acce- sibilidad a los recursos (Gordon, 1980), no se observan diferencias significativas $\left(X^{2}=.226\right.$; $p=.893$ ). Tanto los contextos rurales como los urbanos siguen la misma tendencia, resultando las actuaciones selectivas e indicadas las más habituales.

En tercer lugar, se ha puesto en relación las modalidades de intervención con los contextos rurales y urbanos. En este caso tampoco se han obtenido diferencias estadísticamente significativas $\left(X^{2}=.034 ; p=.855\right)$. En ambos contextos, la modalidad individual/familiar es la más destacada, representando más de la mitad de los recursos de intervención, alcanzando el 68,9 por ciento en el ámbito rural y el 69,9 por ciento en el urbano. 
En último lugar se han estudiado los datos que se refieren a la sistematicidad de las intervenciones. Los resultados muestran que en los dos contextos la amplia mayoría de intervenciones son sistemáticas, el 79,5 por ciento se desarrollan en contexto rural y el 78,2 por ciento se aplican en zonas urbanas, no observándose tampoco diferencias estadísticamente significativas en este caso $\left(X^{2}=.227 ; p=.893\right)$.

Se describen a continuación las relaciones existentes entre el nivel socioeconómico de las zonas y las cuatro variables. En primer lugar, se ha analizado si varía el porcentaje de las intervenciones psicoeducativas que se desarrollan en función del nivel socioeconómico de la zona de aplicación. Se observa que este tipo de recursos se encuentran en mayor proporción en las zonas básicas de Servicios Sociales con nivel socioeconómico más bajo $(41,2$ por ciento). A pesar de ello, las diferencias observadas no son estadísticamente significativas $\left(X^{2}=\right.$ $.306 ; p=.959)$.

Los datos obtenidos manifiestan que generalmente en todas las zonas, independientemente del nivel socioeconómico, las intervenciones predominantes son las selectivas indicadas. No se observan diferencias estadísticamente significativas $\left(X^{2}=1.340 ; p=.969\right)$.

En tercer lugar, los recursos de carácter individual/familiar son los más destacados en la muestra, tanto en los niveles socioeconómicos altos como bajos. En este caso tampoco existen diferencias estadísticamente significativas $\left(X^{2}=.721 ; p=.868\right)$.

Por último y en cuanto a la relación entre el nivel socioeconómico y la sistematicidad de los recursos, los datos muestran que en todas las zonas, independientemente de su nivel socioeconómico, las intervenciones de atención y apoyo familiar sistematizadas son las más representativas, seguidas muy de lejos de las intervenciones sistemáticas de forma parcial. Los recursos no sistemáticos tienen muy poca presencia en todos los casos. No existen diferencias estadísticamente significativas en el grado de estructuración de las intervenciones en función del nivel socioeconómico de la zona de aplicación $\left(X^{2}=1.768 ; p=.940\right)$.

\subsection{Características y condiciones específi- cas de los programas de carácter psicoedu- cativo}

Para analizar la relación entre los requisitos de acceso y el carácter psicoeducativo de las intervenciones se ha seguido también a Gordon (1980). Los resultados de esta relación son muy significativos $\left(X^{2}=13.943 ; p=.01\right)$, reflejando que los recursos psicosociales/psicoeducativos mayoritariamente son de acceso selectivo ( 58,7 por ciento); es decir, que la población puede acceder a ellos si cumplen algún tipo de requisito previo.

En cuanto a la relación entre la modalidad de intervención y el carácter psicoeducativo o no de las intervenciones, los resultados obtenidos son también altamente significativos $\left(X^{2}\right.$ $=148,306 ; p=.000)$. Los recursos psicoeducativos se desarrollan principalmente con modalidad grupal o comunitaria (7,5 por ciento), siendo por el contrario las intervenciones de carácter no psicoeducativo las que se aplican mayoritariamente con una modalidad individual o familiar (96,4por ciento).

Finalmente, con respecto al grado de sistematicidad las pruebas también son estadísticamente significativas $\left(X^{2}=95.521 ; p=.000\right)$. Las intervenciones de carácter psicoeducativo son sistemáticas $(51,4$ por ciento) o lo son parcialmente $(47,7$ por ciento). Sólo un porcentaje muy pequeño de este tipo de recursos no sigue ningún proceso metódico previo.

\section{Discusión}

Los resultados de esta investigación giran en torno a varios bloques. En cuanto a los recursos de intervención, se observa que en situaciones de necesidad se han puesto en marcha actuaciones que tienen que ver principalmente con la promoción y la preservación familiar (carácter psicosocial/psicoeducativo), seguido de intervenciones de apoyo económico, que toman un segundo pero destacado plano.

A partir de ahí, el apoyo en vivienda, empleo y alimentación refuerzan el perfil de la intervención profesional, que apuesta por el empoderamiento, el refuerzo relacional familiar y el favorecimiento del re-equilibrio familiar a través de una protección sustancial.

Se pone de manifiesto el incipiente cambio de perspectiva en las acciones públicas con respecto a la protección familiar, que ya no se limitan a los planteamientos tradicionales y meramente asistencialistas, sino que abogan por una perspectiva más positiva, preventiva y fortalecedora de la intervención familiar (Rodrigo, Máiquez, Martín y Byrne, 2008).

En este sentido, la perspectiva de las fortalezas dentro de la intervención en Trabajo 
Social va tomando cada vez más fuerza, posibilitando la apertura del campo de acción hacia la potenciación individual y social y, por tanto, hacia el desarrollo de los puntos fuertes desde el descubrimiento, la transformación, la certidumbre y el trabajo activo de las personas (De la Paz, 2011).

Las zonas de Diputación contienen un mayor número de recursos que los ayuntamientos, excepto en el caso de la capital. Este resultado muestra la diferencia en la capacidad de atención entre las distintas corporaciones locales, traduciéndose en detrimento de las grandes poblaciones urbanas, donde se encuentran la mayor parte de las familias con hijos, que se alojan fundamentalmente fuera de los ámbitos de la Diputación.

Las intervenciones de atención y apoyo familiar que se llevan a cabo son en su mayoría las mismas en todas las zonas y dispositivos, ya que su aplicación está estipulada en la legislación vigente. Éstas representan más del doble que las intervenciones específicas por zona, que son aquellas no obligatorias por mandato legislativo. Esto refleja una tendencia homogénea a hora de implantar medidas que cubran las necesidades de las familias que se atienden desde los Servicios Sociales comunitarios. En cuanto a las acciones específicas tampoco existe una amplia heterogeneidad.

Por otro lado, y en prueba de la especial vulnerabilidad y riesgo de exclusión social en las que se encuentran las llamadas "zonas de necesidades de transformación social" (ZNTS), también presentan resultados diferentes en el número de intervenciones de apoyo familiar que reciben con respecto al resto, contando con mayor número de acciones específicas adaptadas a las necesidades especiales de su población.

Los dispositivos trabajan principalmente en red con intervenciones intersectoriales que abarcan las problemáticas familiares de forma más integral. De esta lectura se puede deducir que los servicios de atención pública han tenido que abrir sus puertas a colaboraciones externas, no sólo por el beneficio que esto aporta a la mejora de la intervención, sino porque las carencias de recursos ha hecho necesario la alianza entre equipos profesionales que puedan dar respuesta a las nuevas y complejas problemáticas familiares.

Estas necesidades se atienden con el convencimiento de que son prioritarias y urgentes y que no pueden esperar a la burocracia legis- lativa e institucional, poniéndose así en manos de las entidades pertenecientes al Tercer Sector. Estas organizaciones sociales se encuentran en mejor tesitura para atender problemáticas específicas que el sector público que, bien por falta de presupuesto o de capacidad de gestión, es incapaz de absorber de forma eficiente (Buffet, Rubio y Sosvilla, 2013).

Por ello, después de las intervenciones que son comunes en todas las zonas impuestas por imperativos legales o por planes de acción y que, por lo tanto son sistemáticas, están aquellos recursos iniciados con el convencimiento profesional de su necesidad y que cuentan con evidencias derivadas de la experiencia. Estos datos ponen de manifiesto la importante labor profesional que los equipos interdisciplinares que componen los Servicios Sociales comunitarios realizan en el proceso de intervención social y en la calidad de estos servicios.

Los recursos identificados en su mayoría no son de carácter universal, sino que la población que accede a los mismos normalmente presenta alguna característica específica, generalmente un nivel de riesgo medio o alto. Es por ello por lo que el trabajo de estas intervenciones, en su mayoría preventivas, cobran una gran importancia en el ámbito de la Atención Primaria.

Los Servicios Sociales Comunitarios implementan acciones que, en su mayoría, son de modalidad individual/familiar frente a las grupales/comunitarias. En este sentido, hay que destacar que ambos tipos de recursos son ventajosos y necesarios. Por un lado, las intervenciones individuales atienden las necesidades particulares de cada miembro y, por el otro las grupales, también presentes en el análisis aunque en menor número, inciden en las necesidades familiares, tomando como base que la familia es más que la suma de sus miembros. Para conseguir que las intervenciones sean integrales y eficientes es necesario que ambas modalidades se complementen y estén lo más equiparadas posible en cuanto al número de recursos.

El binomio individual/grupal es un mecanismo de acción donde las familias en su conjunto son las destinatarias protagonistas en las intervenciones, seguida de los adultos y los adolescentes (Taylor, 2007, citado en Arranz y Oliva, 2010). De los recursos de apoyo en todos los dispositivos, destacan los destinados a paliar las problemáticas relacionadas con estas dimensiones. 
Las intervenciones de carácter psicosocial/ psicoeducativo son fundamentalmente de acceso no universal, con lo que las familias beneficiarias tienen que ser derivadas por profesionales que han realizado un diagnóstico previo. Los recursos psicoeducativos identificados son fundamentalmente de modalidad grupal/comunitaria y tienen un alto grado de sistematicidad, lo que implica que estas intervenciones cumplen con algunos de los estándares de los programas basados en evidencia (Small, Cooney y O'Connor, 2009).

En este sentido, cabe señalar la importancia de llevar a cabo actuaciones sistematizadas y cuyos resultados sean evaluados. Es necesario hacer una valoración del impacto de la labor profesional proporcionando una retroalimentación que servirá, por un lado, como base para realizar mejoras en las políticas sociales y en el enriquecimiento de la toma de decisiones profesionales y, por otro, para avanzar mediante intervenciones basadas en la evidencia, contribuyendo al establecimiento de criterios de calidad que configuren un servicio de naturaleza análoga al valor individual y social que la familia posee.

Por otra parte, en relación al deficitario desarrollo de los Servicios Sociales comunitarios de Andalucía expuesto (García, Barriga, Ramírez, Zubiría y Velasco, 2016), cabe destacar que el Sistema Público de Servicios Sociales comunitarios tiene suficientes recursos de atención psicosocial/psicoeducativa y de carácter económico, al igual que destacan los de acceso a la información y asesoramiento; pero adolece de otro tipo de intervenciones como son las de ocio y tiempo libre, que parecen olvidadas y son igualmente necesarias, sobre todo en el colectivo de menores. Es necesario incidir en la importancia de una revisión e impulso de las actuaciones lúdicas dirigidas especialmente a adolescentes. Además, los grupos de ayuda mutua y grupos de apoyo no están los suficientemente representados y son intervenciones de sobra conocidas por su necesidad de implantación en todas las zonas y a todos los perfiles familiares (Santana, 2003).

Sin duda, la escasez de este tipo de intervenciones tiene que ver con la situación de crisis existente desde hace casi una década, al ser las primeras que se vieron perjudicadas por los recortes en el gasto social, desestabilizando así la eficiencia de una intervención integral y sistémica. Ante el incremento y complejidad de las demandas actuales, se han realizado interven- ciones con objetivos muy concretos y basados siempre en un tipo específico de prestaciones como asidero principal ante situaciones de desequilibrio, dejando a un lado otras igualmente prioritarias como las dirigidas a la construcción, mejora y enriquecimiento de las interrelaciones sociales (De la Red y Barranco, 2014).

\section{Conclusiones}

En cuanto a los recursos, es necesario resaltar que deben incidir por igual en los tres ejes centrales que desequilibran el sistema familiar en la actualidad: el económico, el interrelacional y el psicosocial. Todo ello desde la apuesta de la adaptación a las necesidades reales, abarcando todas sus dimensiones y en conjunto, utilizando las modalidades de intervención como formas complementarias para ofrecer respuestas más enriquecedoras a las realidades existentes.

De igual forma, el empoderamiento, la autonomía y la autodeterminación individual y colectiva, como dispone la nueva Ley de Servicios Sociales de Andalucía, son elementos de extremo valor que deben ser tenidos en cuenta en todas las implementaciones, especialmente en las dirigidas a las familias en situación de riesgo.

Dentro de estas acciones, hay tener en cuenta que las evaluaciones de las intervenciones genera un conocimiento empírico que dirige acciones eficaces y eficientes basadas en la evidencia científica y en las buenas prácticas profesionales. Por lo tanto, es de vital importancia otorgarle un espacio en el proceso cíclico tanto de la investigación como de la intervención.

Por último, no podemos olvidar que el contexto de intervención local debe tenerse en cuenta como pieza clave en la toma de decisiones a nivel provincial, bajo los principios rectores del Sistema, en especial, el de proximidad.

En este sentido, hubiera sido interesante realizar el análisis de la percepción de las familias usuarias con respecto a los recursos de los que disponen. Otra de las limitaciones se encuentra dentro del proceso de trabajo de campo, y tiene que ver con el análisis del Tercer Sector. Una comparativa entre los dos estudios ofrecería conclusiones más amplias sobre la forma en que la atención familiar ha cambiado tras la crisis económica. También 
habría sido interesante abordar el estudio de los dispositivos de atención especializada, ampliando la fotografía del mapa de recursos para familias con hijos menores a cargo.

En definitiva, objetivos como lograr intervenciones basadas en la evidencia y apostar por el feed-back constante entre intervención e investigación son indispensables para alcanzar la excelencia en la intervención familiar y en las actuaciones que desarrollan el Trabajo Social y demás profesionales afines al ámbito de lo so- cial, para lograr la cohesión y el cambio social. Un objetivo común tan prioritario como complejo en el marco de la actual configuración estructural de la sociedad a la que llamamos postmoderna y que está acompañada de cambios en la familia y de su entorno. Transformaciones que dibujan otro panorama de necesidades más holístico, multidimensional y heterogéneo $\mathrm{y}$ que, por tanto, necesitan de intervenciones que al mismo nivel satisfagan de forma integral estas nuevas necesidades coyunturales.

\section{Referencias bibliográficas}

Arranz, E. y Oliva, A. (coords.) (2010). Desarrollo psicológico en las nuevas estructuras familiares. Madrid: Pirámide.

Beck, U., Giddens, A. y Lash, E. (eds.) (1996). Reflexive Modernisierung. EineKontroverse. Frankfurt: Suhrkamp. Traducido por Orestes Sandoval López (1999). Modernización reflexiva. Archplus, 146. Disponible en: http://www.criterios.es/pdf/archplusbeckmoder.pdf

Buffet, P., Rubio, J.J. y Sosvilla, S. (2013). El tercer sector social en España: atributos y características del mecenazgo y la filantropía. Información Comercial Española. Revista de Economía, 872, 79-91.

Casado, D. (2008). Los servicios sociales: carencias relativas a las situaciones de pobreza, marginación social, exclusión y desigualdades. En: VI Informe sobre Exclusión y Desarrollo social en España (pp.145156). Madrid: Fundación Foessa.

De la Paz, P. (2011). La intervención en Trabajo Social desde la perspectiva de las fortalezas. Cuadernos De Trabajo Social, 24, 155-163. doi:10.5209/rev_CUTS.2011.v24.36865

De la Red, N. y Barranco, C. (2014). Trabajo Social y participación en las políticas sociales. Azarbe. Revista Internacional de Trabajo Social y Bienestar, 3, 39-45.

Flaquer, L. (2000). Las políticas familiares en una perspectiva comparada. Barcelona: Fundación La Caixa.

Flaquer, L. (2004). La articulación entre familia y el estado de bienestar en los países de la Europa del sur. Papers Revista de Sociología, 73, 27-58.

Flaquer, L. (2006). Familia y políticas públicas. En: A.M. Morales Moreno y J.M. Miquel (dirs.), Derecho, sociedad y familia: cambio y continuidad (pp. 229-239). Madrid: Facultad de Derecho de la Universidad Autónoma de Madrid.

Garcés, A. (2012). Los nuevos retos de los servicios sociales en España. Madrid: Epraxis.

García, G., Barriga, L., Ramírez, J.M., Zubiría, A. y Velasco, L. (2016). Índice de Desarrollo de los Servicios Sociales 2015. Madrid: Asociación Estatal de Directores y Gerentes en Servicios Sociales.

Gordon, T. (1980). Parent Effectiveness Training. A preventive program and its effects on families. En: M.J. Fine (ed.), Handbook on Parent Education (pp.101-121). Nueva York: Academic Press.

Hamzaoui, M. (2005). Trabajo Social Territorializado. Las transformaciones de la acción pública en la intervención social. Valencia: NAU Libres.

Instituto Nacional de Estadística.. (2016). Encuesta de Condiciones de Vida (ECV). Año 2015 (resultados definitivos). Nota de Prensa del 24 de mayo de 2016. Recuperado de: http://www.ine.es/prensa/np969. pdf

Lima, A.I. (coord.) (2016). II Informe sobre los Servicios Sociales en España (ISSE). Madrid: Consejo General del Trabajo Social.

Menéndez, S., Arenas, A.V., Pérez, J. y Lorence, B. (2012). Madres usuarias de servicios de preservación familiar: perfil sociodemográfico y evolución. Cuadernos de Trabajo Social, 25(1), 193-203. doi: http:// dx.doi.org/10.5209/rev_CUTS.2012.v25.n1.38444

Montagut, T. (2008). Política Social. Una introducción. Barcelona: Ariel Sociología.

Moreno, L. (2002). Bienestar mediterráneo y supermujeres. Revista Española de Sociología, 2, 41-56.

Naldini, M. (2003). The Family in the Mediterranean Welfare States. London \& Portland Or.: Frank Cass.

Navarro, V. y Clua-Losada, M. (dirs.) (2012). El impacto de la crisis en las familias y en la infancia. Observatorio Social de España. Barcelona: Ariel sociología. 
Rodrigo, M.J., Máiquez, M.L. y Martín-Quintana, JC. (2010). Parentalidad positiva y políticas locales de apoyo a las familias. Madrid: Federación Española de Municipios y Provincias.

Rodrigo, M.J., Máiquez, M.L., Martín, J.C. y Byrne, S. (2008). Preservación familiar. Un enfoque positivo para la intervención con familias. Madrid: Pirámide.

Rodrigo, M.J. y Palacios, J. (1998). Familia y desarrollo humano. Madrid: Alianza Editorial.

Ruiz, C. (1999). La familia y su implicación en el desarrollo infantil. Revista Complutense de Educación, 10(1), 289-304.

Santana, I. (2003). El apoyo formal a la familia como cuidadora natural. Portularia, 3, 117-135.

Small, A.S., Cooney, M.C. y O'Connor, C. (2009). Evidence-Informed Program Improvement: Using Principles of Effectiveness to Enhance the Quality and Impact of Family-Based Prevention Programs. Family Relations, 58, 1-13. 